\title{
An Enhanced and Automatic Skin Cancer Detection using K-Mean and PSO Technique
}

\author{
Ramandeep Kaur, Gagandeep, Parveen Kumar, Geetanjali Babbar
}

\begin{abstract}
Scientists have been trying to implement traditional methods around the world, particularly in developing countries, to reduce the death rate of skin cancer in humans. The scientific term is named as melanoma. But this effort always working hard as the system is costly, the low availability of experts and the conventional telemedicine. There are three types of skin cancer: basal cell cancer (BCC), squamous cell cancer, and melanoma. More than $90 \%$ of human is affected by ultraviolet $(U V)$ radiation exposed to the sun. In this research, a skin cancer detection system $(B C C)$ is designed in MATLAB. The images going to different processes such as Pre processing, feature extraction and classification. In pre-processing K-mean clustering is applied to determine the foreground and background of an image, since some part of background appear in the image after K-mean. Therefore, to resolve this problem Particle Swarm optimization (PSO) is applied. The segmented image features are extracted using Speed Up Robust Features (SURF), this helps to enhance the quality of the image. The Artificial neural network $(A N N)$ is trained on the basis of these extracted features. To determine the efficiency of the system, the images are tested and performance parameters are measured. The detection accuracy determined by this model is about 98.75 is obtained.
\end{abstract}

Index Terms: Skin Cancer, K-mean, PSO, SURF, ANN

\section{INTRODUCTION}

Skin cancers are the type of cancers which is originated from the human skin. Skin cancer is associated with the development of abnormal cells that have the capability to spread in the entire body. The skin cancer is mainly categorized into three different types namely; Basal Cellular Cancer (BCC), Squamous Cell Skin Cancer (SCC), and melanoma [1]. Melanoma, also known as malignant among these species, and is a type of cancer that occurs from cells containing pigment, also named as melanocytes. Melanomas are commonly found in the skin but rarely appear in the mouth, and in the eye. Women generally find this type of cancer on their feet, whereas in men there are found on the back[2].Most of the time these start from a molewith a change in size, irregular edges, colour change, itching, or changes in skin colour.

The utmost cause of melanoma is the effect of UV in low-level skin pigment. The resources of UV might be from

\section{Revised Manuscript Received on June 15, 2019.}

Ramandeep Kaur, Computer Science and Engineering ,Chandigarh Engineering College, Landran, Mohali, India.

Dr.Gagandeep Computer Science and Engineering ,Chandigarh Engineering College, Landran, Mohali, India.

Parveen Kumar, Computer Science and Engineering ,Chandigarh Engineering College, Landran, Mohali, India.

Geetanjali Babbar, Computer Science and Engineering ,Chandigarh

Engineering College, Landran, Mohali ,India. sun rays like as tanning on the human skin. But $25 \%$ of the patient are suffered from skin cancer due to the formation of the mole [3]. The person with multiple models, the background of the patient which is being affected by skin cancer and having the less immune system have also increased the factor of risk. The doctors diagnose skin cancer through the biopsy process, and analysis of any skin lesions with signs of potential cancer has been performed[4].

About 232,000 people around the world, who are affected by skin cancer,appeared in 2012 . About 59,800 people were died that resulted in an active disease of about 3.1 million in 2015. Also in Australia and New Zealand have the highest rates of melanoma in the world [5]. There are high rates in Northern Europe and North America, and Europe is relatively low compared to Asia, Africa and other Latin American countries. Melanoma is more common in gents than Females. Melanoma has become more popular in areas with white population since the 1960s [6].

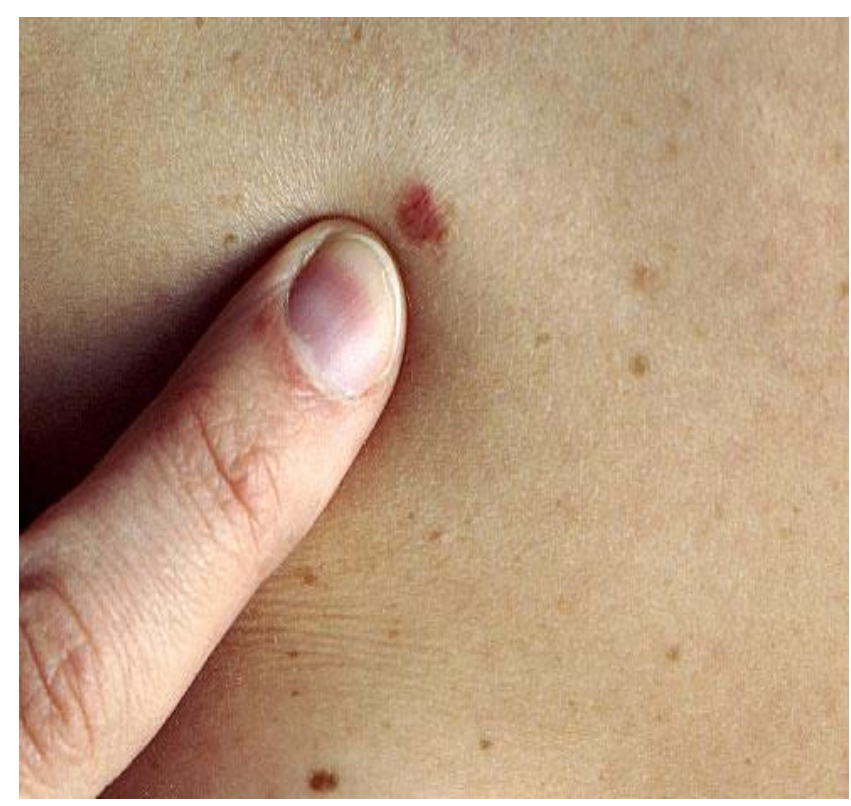

Fig 1: Skin Cancer

There are a different technique in the detection of cancer among them Dynamic thermal imaging (DTI) is the most common technique due to its rapid, accurate and non-imaging method for detection of skin cancer [7].

In this, there is a cooling of the lesion and thermal recovery is a monitor with the help of infrared imaging. After that thermal recovery curves of the suspected lesions are then utilized in the context of regular time detection theory for the formation of an 
optical statistical decision rule due to this the sensitivity of the method is to be maximum for every prescribed false-alarm probability [8].This research is helpful in those areas where the high qualified doctors are not available like in the rural area. Therefore, this system helps people to save their lives by detecting basal cell carcinomas at their earlier described in the following section.

\section{Related Work}

Sheha, M. et al. $(9,2012)$ have employed an automated method for the diagnosis of melanoma which has been implemented to a group of dermoscopy images. The resulting features are based on the use of the gray level synchronization matrix (GLCM) and Multilayer perceptron classifier (MLP) to differentiate the Melanocytic Nerve from Malignant melanoma. The MLP classifier has been utilized in two different ways in the training as well as in the testing process: an intelligent MLP and conventional MLP. From the test results, it has been found that the examination of melanocytic through texture analysis process is a better method.The first technique, the Automation replicator is faster, but secondly, the default scheme provides $100 \%$ accuracy for the training kit and $92 \%$ for the test kit.

Masood et al. $(10,2013)$ demonstrated a statistics analysis from the most important applications reported so far. The results from different classifiers have been studied to examine the skin lesion as well as described the outcomes. They offered a framework for the comparative evaluation of diagnostic models of skin cancer and a review of results based on these models. These Image-based computer-assisted diagnostic systems have important potential for screening and detection of malignant melanoma at an early stage.

Åberg. P et al $(11,2005)$ proposed and compare the detection accuracy of the designed skin cancer model by employing impedance spectra. The presented work has utilized two types of electrodes and obtained the signals without optimization of power.Using two electrode systems, 99 benign neves, 28 impedance of BCC and 13 Malignant melanoma (MM) have been measured. The classification of lesions has been used with Fisher's linear discriminant and has been evaluated using the cross-validation of technological sensitivity and specifications.

Calin. $M$ et al. $(12,2013)$ have employed the most inspected optical diagnostic scheme named as optical coherence tomography, fluorescence spectrometry, reflectance spectrometry, Raman spectroscopy, and confocal microscopy. Various methods have been used to assess the performance of this technique in the preoperative and postoperative diagnostics of skin cancers, using specific keywords and accurate access and exclusion criteria. It has been concluded that the optical coherence tomography showed promising results in evaluating deep margins of skin tumours and inflammatory skin diseases, but less effective in distinguishing premalignant malignant lesions. stage. The work performed by various authors in this field is

\section{PROPOSED WORK}

The flow of work, which is incorporated in Digital Image processing is represented in the form of block diagram as depicted in figure 2.

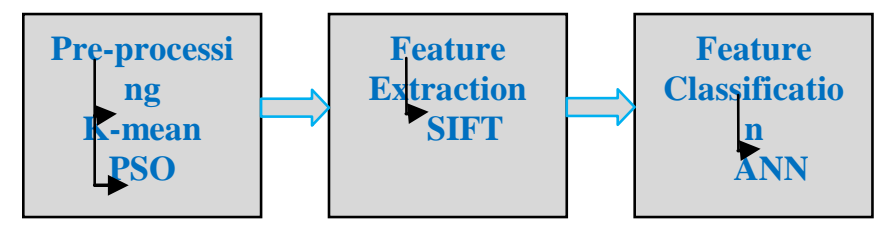

Figure 2: Block Diagram of proposed Skin Cancer Detection System

\section{A. Pre-processing}

Pre-processing is applied to the image in order to increase the quality of an image as these images have been captured in different lighting conditions, therefore, needs to be preprocessed. In this research, the separation of foreground and background have been performed using K-mean technique as shown in figure 3. It is a partitioning method, which groups images in such a way so that the images in a particular group contain minimum variations among them. If the variance within the group is minimized, it provides a high feature segmentation image.

1. Initialize two centroids randomly within an image.

2. Determine the distance between the every pixel element from the centroid and allocate each pixel to its nearby centroid.

3. The centroid position can be reallocated by measuring the mean value of a similar group.

4. Reallocate the novel position of the centroid within the same image.

5. The case when the centroid value changes repeat the process until a stable centroid is obtained.

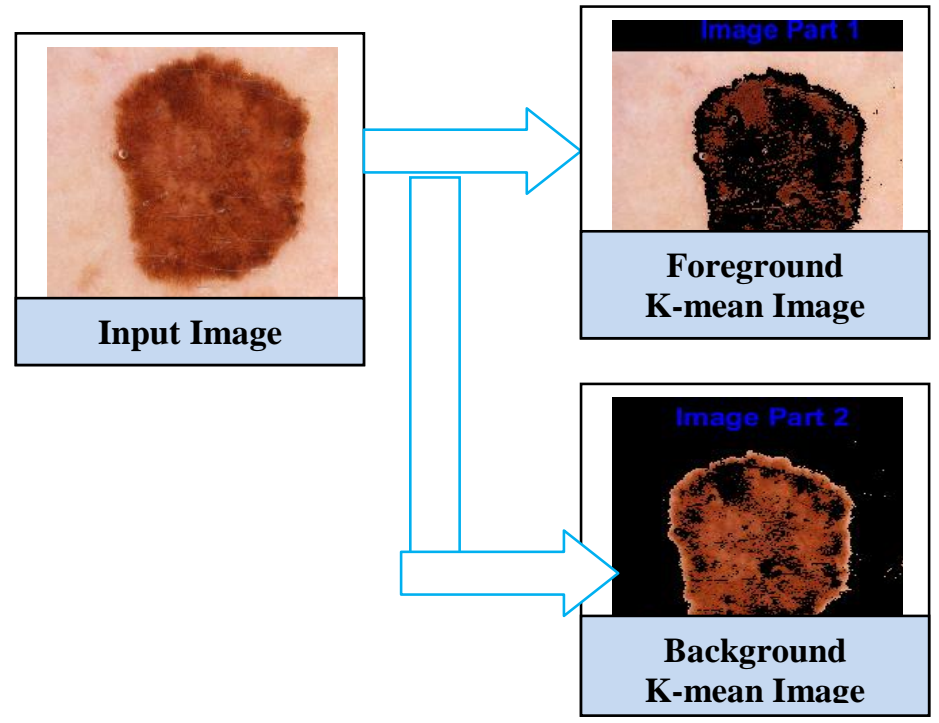

Figure 3: K-mean Clustered Image

\section{B. PSO Algorithm}

To enhance the features of the image an optimization technique named as PSO has been applied. PSO is a swarm inspired algorithm used to resolve the complex problem. In PSO, a threshold value is defined on the basis 
of which the space with background has been replaced with the foreground so that the skin cancer can be detected properly. The image obtained after PSO is shown in figure 4.

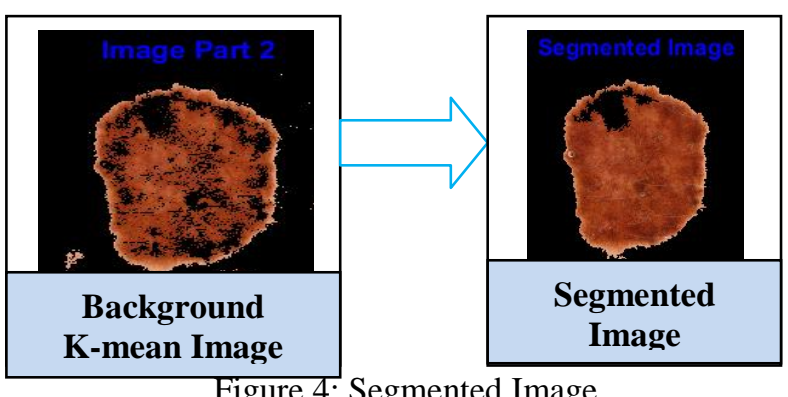

As shown in figure 4, The black colour (background) of an image is replaced by the pixels of the same test image so that the classification can be done with high accuracy.

\section{Feature Extraction (SURF)}

The aim of using feature extraction is to reduce the size as well as to find the appropriate features from the image. In this research, SURF is used as a feature descriptor to extract features from the segmented image. This algorithm works in three different phases such as detection of interest point, description of local neighbourhood and matching. The desired points are extracted as per the Hessian matrix.

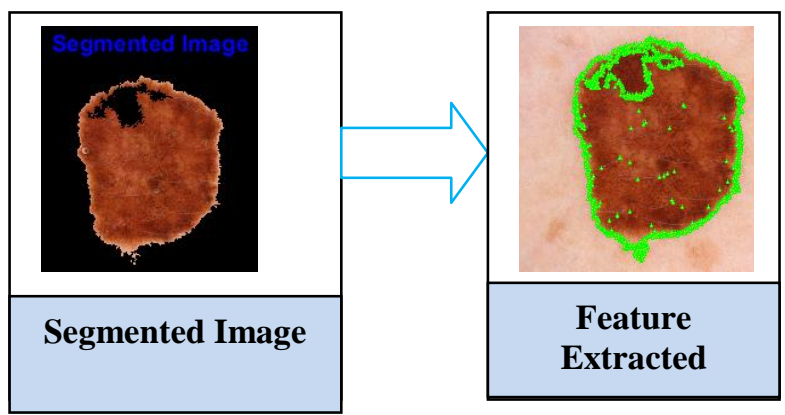

Figure 5: SURF Extracted Features

\section{Classification (ANN)}

During the training section, the segmented images are used as an input to the classifier to train the system with high accuracy. The training of ANN during experimental work is shown in the figure 6.

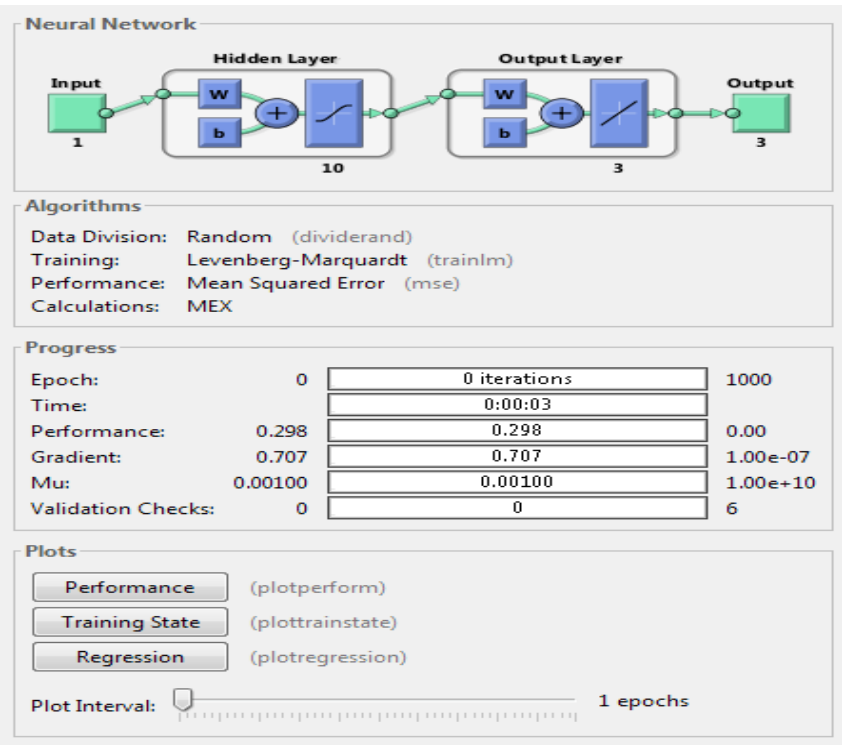

Figure 6: ANN Training Structure

The training of the designed "Skin cancer detection system" using ANN is shown in figure 6. The extracted features are provided as an input to the ANN layer, which is then modified using a number of neurons so that desired results can be obtained. The trained structure is stored into the database and during testing, the matching is performed to identify the skin cancer. If the features are matched with the stored data, then the lesion on the skin is affected by cancer otherwise considered as a normal node.

\section{Results and Discussion}

To measure the efficiency of the designed system, the performance has been determined in terms of different parameters such as TPR, FPR, Precision and Accuracy. The parameter's value examined for 5 number of different test samples are listed in the table I.

Table I: Computed Parameters

\begin{tabular}{|c|c|c|c|c|}
\hline $\begin{array}{c}\text { Number } \\
\text { of Test } \\
\text { Samples }\end{array}$ & TPR & FPR & Precision & Accuracy \\
\hline $\mathbf{1}$ & 0.957 & 0.092 & 0.963 & 99.17 \\
\hline $\mathbf{2}$ & 0.945 & 0.074 & 0.973 & 98.18 \\
\hline $\mathbf{3}$ & 0.931 & 0.068 & 0.984 & 98.18 \\
\hline $\mathbf{4}$ & 0.939 & 0.079 & 0.988 & 98.23 \\
\hline $\mathbf{5}$ & 0.973 & 0.046 & 0.951 & 99.83 \\
\hline
\end{tabular}




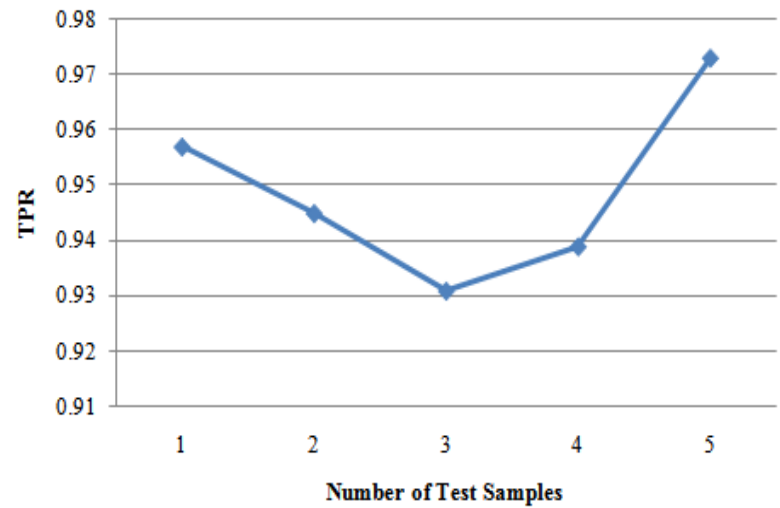

Figure 7: TPR

The TPR of the proposed work is shown in figure 7. From the figure it is clear that the TPR for five numbers of samples increases. It is minimum for sample 1 and maximum for sample 5 .

The maximum value of TPR represents that the samples are detected with higher accuracy. The average rate determined for five numbers of samples is 0.949 .

The graphical representation of the false positive rate for the proposed model is shown in figure 8 . The average value of FPR determined for five different test samples is 0.071 , which is very much less compared to the TPR rate, this indicates that the design model providesa false result for very few samples. That is out of 100 test samples 93 samples are detected accurately.

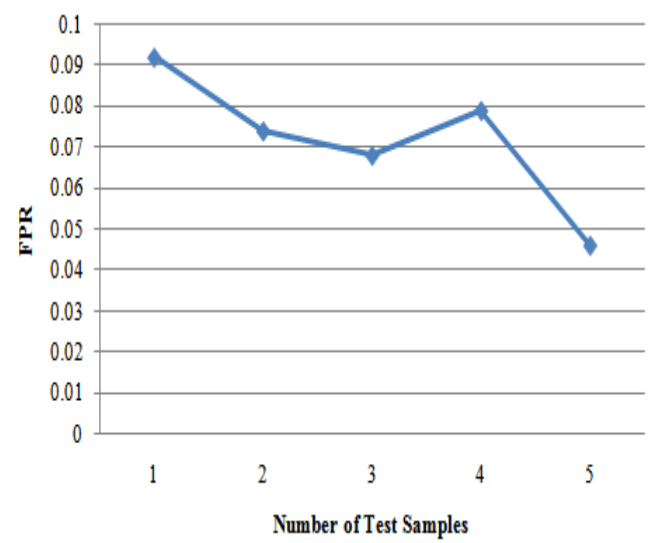

Figure 8: FPR

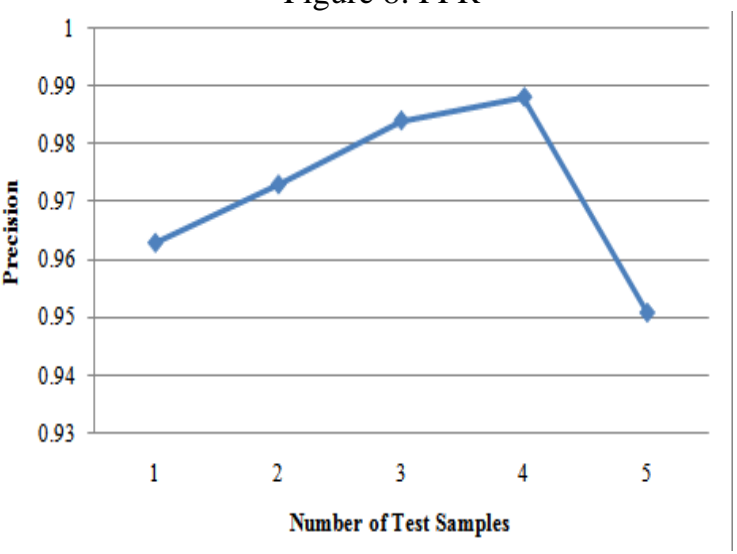

Figure 9 Precision
The precision values measured for the five number of skin cancer samples are shown in figure 9. The average value measured for five samples is 0.9718 .

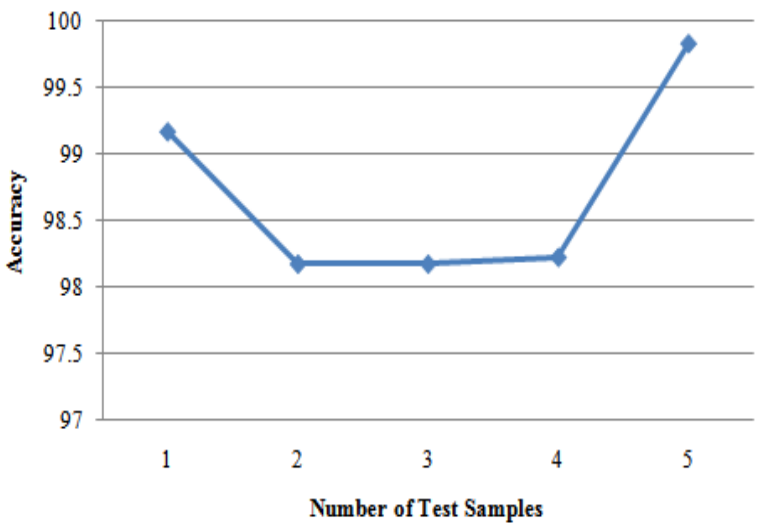

Figure 10 Accuracy

The accuracy or the detection rate to detect skin cancer accurately is represented in figure 10. The higher the accuracy rate more efficiency, the system can detect skin cancer. In this work, the mean of detection accuracy determined for five different samples is 98.718 .

\section{A. Comparison of proposed work with existing work}

To compare the performance results, the paper of Kharazmi et al., which was published in the year 2019 in IEEE journal of biomedical and health informatics have been considered. The authors have worked to detect skin lesion using the concept of K-mean clustering. The values examined by the authors are depicted in table II.

Table II Comparison Results

\begin{tabular}{|c|c|c|c|c|}
\hline & TPR & FPR & Precision & Accuracy \\
\hline $\begin{array}{c}\text { Kharazmi } \\
\text { et al [13] }\end{array}$ & 0.933 & 0.100 & 0.952 & 0.922 \\
\hline $\begin{array}{c}\text { Proposed } \\
\text { Work }\end{array}$ & 0.949 & 0.071 & 0.971 & 0.987 \\
\hline
\end{tabular}

\section{Comparison of TPR \& FPR}

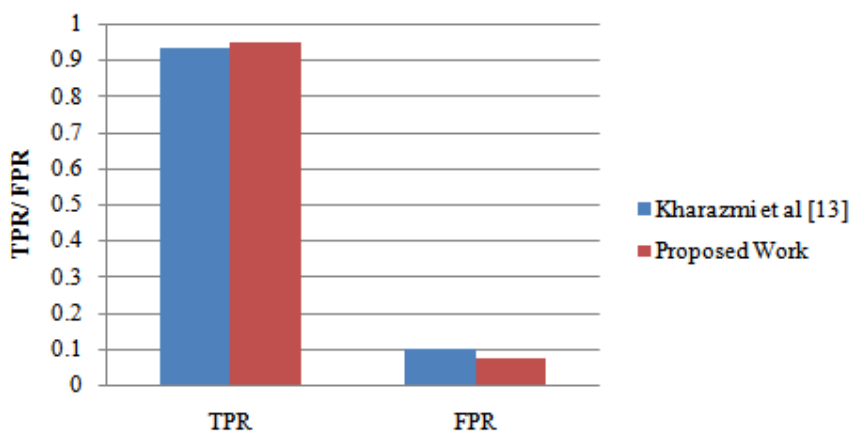

Figure 11: Comparison of TPR \&FPR

The comparison of TPR and FPR of the proposed work with Kharazmi et al [13] has been presented. From the figure 11 , it is clear that the TPR of the proposed is higher about $1.71 \%$ than the existing work. The FPR of the proposed work has been reduced by $29 \%$ from the Kharazmi et al [13]. 


\section{Comparison of Precision}

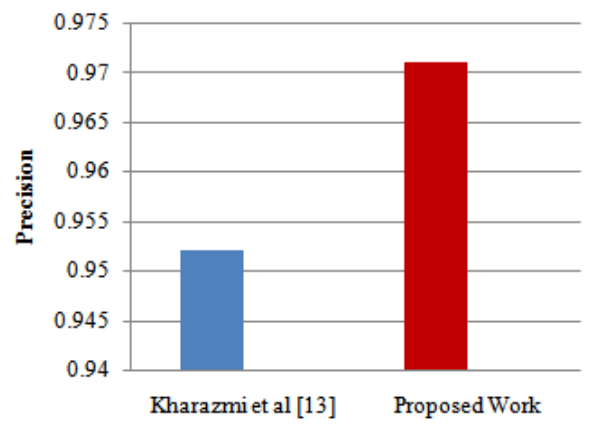

- Kharazmi et al [13] - Proposed Work

Figure 12: Comparison of Precision

The precision comparison of the proposed work with the existing work is represented in figure 12 . The precision of the proposed work is enhanced by $2 \%$ compared to the work performed by Kharazmi et al [13]. This is due to the proper selection of pixels which is done by the optimization algorithm.

\section{Comparison of Accuracy}

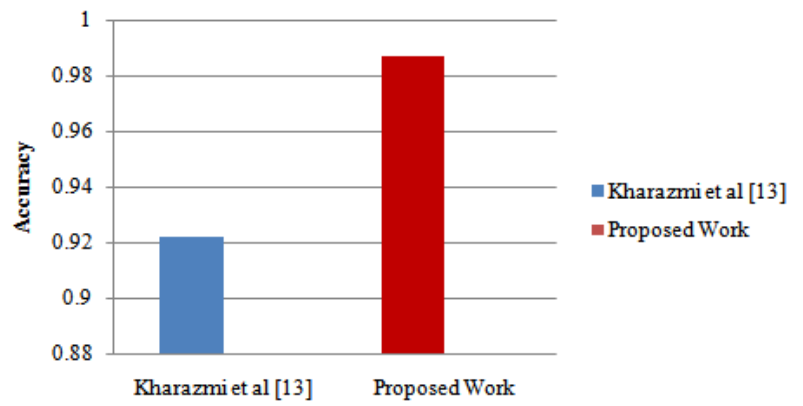

Figure 13: Comparison of Accuracy

The comparison of accuracy is shown in figure 13, From the figure, it is clear that the detection accuracy of the proposed work is higher than the P. Kharazmi et al [13]. The improvement in the accuracy of about $7.05 \%$ has been obtained.

\section{CONCLUSION}

The diagnosis of skin lesions has been performed on a complete test image. It was found to work well with an accuracy rate of $98.7 \%$. Automatic diagnosis is very simple, practical and attainable through well-defined segmentation (K-mean \& PSO), feature extraction (SURF) and classification techniques (ANN). The utilization of K-mean with PSO helps to enhance the quality of the image and hence results in increased detection accuracy. At last, the comparison between proposed and existing work performed by Kharazmi et al has been represented and observed that the TPR, precision, Accuracy is higher compared to the existing work. About $7.05 \%$ enhancement in the detection of skin cancer has been obtained compared to the existing work.

\section{REFERENCES}

1. N. C.Codella, D.Gutman, M. E.Celebi, B.Helba, M. A.Marchetti, S. W.Dusza, ... \& A.Halpern, "Skin lesion analysis toward melanoma detection,"A challenge at the 2017 international symposium on biomedical imaging (isbi), hosted by the international skin imaging collaboration (isic). In 2018 IEEE 15th International Symposium on Biomedical Imaging, 2018, pp. 168-172.

2. A. C.Geller, R. R.Keske, S.Haneuse, J. A.Davine, K. M.Emmons, C. L.Daniel, ... \& L. L.Robison, "Skin cancer early detection practices among adult survivors of childhood cancer treated with radiation," Journal of Investigative Dermatology, 2019.

3. A.Mirbeik-Sabzevari, Li, S., Garay, E., Nguyen, H. T., Wang, H., \& Tavassolian, N, "Synthetic ultra-high-resolution millimeter-wave imaging for skin cancer detection," IEEE Transactions on Biomedical Engineering, 2019, vol. 66, no. 1, pp.61-71.

4. A.Dascalu, \& E. O. David, "Skin cancer detection by deep learning and sound analysis algorithms: A prospective clinical study of an elementary dermoscope," EBioMedicine, 2019.

5. A. F.Jerant, J. T.Johnson, C.Demastes Sheridan,\& T. J.Caffrey, "Early detection and treatment of skin cancer. American family physician," 2000, vol. 62, no. 2 .

6. R. J.Friedman, D. S.Rigel,\& A. W.Kopf, "Early detection of malignant melanoma: the role of physician examination and self-examination of the skin,"CA: a cancer journal for clinicians, 1985, vol. 35, no.3, pp.130-151.

7. M. A. H.Bhuiyan, I.Azad,\& K. Uddin, "Image processing for skin cancer features extraction," International Journal of Scientific \& Engineering Research, 2013, vol. 4, no. 2, pp.1-6.

8. J.Choi, J.Choo, H.Chung, D. G.Gweon, J.,Park, H. J.Kim, ... \& Oh, C. $\mathrm{H}$, "Direct observation of spectral differences between normal and basal cell carcinoma (BCC) tissues using confocal Raman microscopy," Biopolymers: Original Research on Biomolecules, 2005, vol. 77, no.5, pp. 264-272.

9. M. A.Sheha, M. S.Mabrouk, \& A.Sharawy, "Automatic detection of melanoma skin cancer using texture analysis," International Journal of Computer Applications, , 2012.

10. A.Masood,\& A.Ali Al-Jumaily, "Computer aided diagnostic support system for skin cancer: a review of techniques algorithms," International journal of biomedical imaging, 2013.

11. P.Åberg, P.Geladi, I.Nicander, J.Hansson, U.Holmgren,\& S.Ollmar, "Non-invasive and microinvasive electrical impedance spectra of skin cancer-a comparison between two techniques," Skin research and technology, 2005, 11(4), 281-286.

12. M. A.Calin, S. V.Parasca, R.Savastru, M. R.Calin,\& S.Dontu,, "Optical techniques for the noninvasive diagnosis of skin cancer," Journal of cancer research and clinical oncology,2013, vol. 139, no.7, pp.1083-1104.

13. P.Kharazmi, M. I.AlJasser, Lui, H., Wang, Z. J., \& Lee, T. K, "Automated detection and segmentation of vascular structures of skin lesions seen in Dermoscopy, with an application to basal cell carcinoma classification," IEEE journal of biomedical and health informatics, 2016, vol.21, no. 6, pp.1675-1684. 


\section{AUTHORS PROFILE}

Ramandeep kaur received my B.Tech degree in Computer Science And Engineeering From RAYAT BAHRA GROUP OF INSTITUTIONS, MOHALI, INDIA in 2017. I am Currently pursuing $\mathrm{M}$.TECH in computer science and engineering at Chandigarh Engineering College (CEC),Landran, Mohali, India. Her Research Interest includes medical image processing.

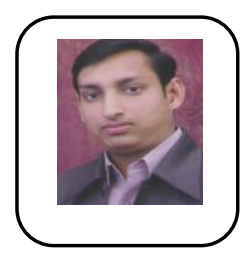

Dr. Gagandeep received his Bachelor's degree in Computer Science and Engineering from Punjab Technical University, Jalandhar, Punjab, India in 2002, M.E. degree in Computer Science and Engineering from PEC University of Technology, Chandigarh, India, in 2005 and Ph.D. degree in Computer Engineering from Panjabi university, Patiala, India, in 2017. Presently, he works in Department of Computer Science \& Engineering, Chandigarh Engineering College (CEC), Landran, Mohali, India as faculty member and has several years work experience in the areas of teaching. $\mathrm{He}$ has published more than 40 research papers in leading journals, conference proceedings and books including Elsevier, IEEE, Taylor $\mathrm{n}$ Francis and Springer and also holds 23 patents. He is also life member of several societies, received prestigious Academia award from CSI consecutively in 2011 \& 2012 and also received ISTE best teacher award in 2016. His current research interests include medical image processing, object detection, semantic retrieval, deep learning.

Geetanjali Babbar,Working as an Assistant Professor in Chandigarh Engineering College, Landran, Pursuing Ph.D in Computer Science, M.Tech in Computer Science, 16 national/international publications, Life time member of Indian Society for Technical Education. I have 15 years of teaching experience in CEC Landran. I have been awarded for best teacher in computer science department of CEC Landran. My research work is in the area of Machine Learning. 